\title{
The Influence of Metabolic Factors for Nonalcoholic Fatty Liver Disease in Women
}

\author{
Goh Eun Chung, Jeong Yoon Yim, Donghee Kim, Seon Hee Lim, \\ Jong In Yang, Young Sun Kim, Sun Young Yang, Min-Sun Kwak, \\ Joo Sung Kim, and Sang-Heon Cho \\ Department of Internal Medicine, Healthcare Research Institute, Gangnam Healthcare Center, Seoul National University Hospital, \\ Seoul, Republic of Korea \\ Correspondence should be addressed to Jeong Yoon Yim; yjy@snuh.org
}

Received 23 January 2015; Revised 27 March 2015; Accepted 29 March 2015

Academic Editor: Luca Miele

Copyright (C) 2015 Goh Eun Chung et al. This is an open access article distributed under the Creative Commons Attribution License, which permits unrestricted use, distribution, and reproduction in any medium, provided the original work is properly cited.

Background/Aims. Women after menopause have increased insulin resistance and visceral fat, which may increase the prevalence of nonalcoholic fatty liver disease (NAFLD). However, the pathogenesis of NAFLD in women has not been clearly defined. In this study, we aimed to determine the risk factors for NAFLD in women. Methods. A retrospective cohort study was conducted. Women who underwent abdominal ultrasonography and blood sampling for routine health check-ups were recruited. Results. Among 1,423 subjects, 695 women $(48.9 \%)$ were in a menopausal state. The prevalence of NAFLD was higher in postmenopausal women than in premenopausal women $(27.2 \%$ versus $14.4 \%, P<0.001)$. In premenopausal women, low HDL-cholesterol, central obesity, and homeostasis model assessment-estimated insulin resistance showed a significant association with the increased risk of NAFLD in multivariate analysis. In postmenopausal women, the presence of diabetes, triglyceridemia, and central obesity showed a significant association with the risk of NAFLD. The presence of menopause and hormone replacement therapy in postmenopausal women were not risk factors for NAFLD. Conclusions. Our findings showed different metabolic factors for NAFLD in pre- and postmenopausal women. However, the key issues are the same: central obesity and insulin resistance. These results reemphasize the importance of metabolic factors irrespective of menopausal status in the pathogenesis of NAFLD in women.

\section{Introduction}

Nonalcoholic fatty liver disease (NAFLD) is the leading cause of chronic liver disease, with increasing in prevalence up to $20-30 \%$ worldwide [1]. It is characterized by the accumulation of fat that is more than $5 \%$ of the hepatocytes in the liver [2], which encompasses various conditions ranging from simple steatosis, nonalcoholic steatohepatitis, to cirrhosis [3]. NAFLD is considered to be a hepatic manifestation of metabolic syndrome (MS), because the central pathogenesis of NAFLD is insulin resistance [4]. NAFLD is closely linked to variable components of MS such as type 2 diabetes, dyslipidemia, and central obesity $[5,6]$.

Women after menopause have increased insulin resistance and visceral abdominal fat, which are risk factors for the development of NAFLD [7]. Previous studies have indicated that gender differences and menopausal status may influence the prevalence and severity of NAFLD, suggesting an association with hepatic steatosis [8-11]. A recent study including patients with histological diagnosis of nonalcoholic steatohepatitis found that postmenopausal women as well as men have an increased risk for advanced fibrosis compared to premenopausal women [11]. NAFLD is more prevalent in postmenopausal women and women with polycystic ovary syndrome than those in a premenopausal state, suggesting a protective role of estrogen against NAFLD [12]. In a doubleblind, randomized controlled study, hormone replacement therapy was associated with liver enzyme levels in women with type 2 diabetes [13]. However, the pathogenesis of NAFLD in women has not been clearly defined, and there is not much information about NAFLD and menopause or hormone replacement therapy, especially in population based studies. Therefore, the aim of this study was to determine the risk factors for NAFLD in women. 


\section{Patients and Methods}

2.1. Study Population. A retrospective cohort study was conducted to evaluate the risk factors for NAFLD in women. Women who underwent abdominal ultrasonography (US) and blood samplings at the Seoul National University Hospital Gangnam Healthcare Center, Seoul, Republic of Korea, for routine health check-ups in 2010 were recruited. Most of the study population paid voluntarily for their health checkups, and some of them were supported by their employer. Among 1,720 subjects, 57 subjects were positive for hepatitis B virus, 22 were positive for hepatitis $C$ virus, 90 had significant alcohol consumption (>20 g/day), 2 had a history of chronic liver disease, and 126 had a past medical history of cancer and these subjects were excluded from the study. Finally, 1,423 subjects met the inclusion criteria. This study was approved by the Institutional Review Board of the Seoul National University Hospital with a waiver of informed consent.

2.2. Clinical and Laboratory Assessments. Each subject completed a past medical history questionnaire including the status of menopause and history of hormone replacement therapy. Women were considered menopausal if menstruation periods had stopped over 12 consecutive months previously. All subjects participated in anthropometric assessment and laboratory and radiologic tests on the same day. Body weight and height were measured using a digital scale, and body mass index (BMI) was calculated by dividing weight $(\mathrm{kg})$ by the square of height $\left(\mathrm{m}^{2}\right)$. Waist circumference was measured at the midpoint between the lower costal margin and the anterior superior iliac crest by a well-trained person using a tape measure. Systolic blood pressure and diastolic blood pressure were measured twice, and the mean values were reported. The presence of hypertension was defined as having a systolic blood pressure $\geq 140 \mathrm{mmHg}$ or diastolic blood pressure $\geq 90 \mathrm{mmHg}$ or use of antihypertensive medication. Current smokers were defined as having smoked at least 1 cigarette/day during the previous year. Former smokers were defined as prior regular cigarette smoking [14].

The laboratory tests included serum alanine aminotransferase (ALT), aspartate aminotransferase (AST), gammaglutamyl transpeptidase (GGT), total cholesterol, triglycerides, high density lipoprotein (HDL) cholesterol, low density lipoprotein (LDL) cholesterol fasting glucose, hepatitis $\mathrm{B}$ surface antigen, and antibody to hepatitis $\mathrm{C}$ virus. Blood samples were collected before 10:00 am after a $12 \mathrm{~h}$ overnight fast. All laboratory tests were carried out using standard laboratory methods. Homeostasis model assessment-estimated insulin resistance (HOMA) was used to evaluate insulin resistance and was obtained by multiplying the fasting insulin by the fasting glucose. The presence of diabetes mellitus was defined as either a fasting serum glucose $\geq 126 \mathrm{mg} / \mathrm{dL}$ or use of antidiabetic medication.

MS was diagnosed when three or more of the five components were present, that is, (1) central obesity (waist circumference as defined by the Regional Office for the Western Pacific Region of the World Health Organization criteria, $>90 \mathrm{~cm}$ (men) or $>80 \mathrm{~cm}$ (women)); (2) a triglyceride level $\geq 150 \mathrm{mg} / \mathrm{dL}$; (3) HDL-C $<40 \mathrm{mg} / \mathrm{dL}$ (men) or $<50 \mathrm{mg} / \mathrm{dL}$ (women); (4) fasting glucose $\geq 100 \mathrm{mg} / \mathrm{dL}$ or treatment for diabetes; (5) arterial pressure $\geq 130 / 85 \mathrm{mmHg}$ or treatment for hypertension [15].

2.3. NAFLD Assessments. NAFLD was defined as the presence of fatty liver disease as determined by US with absence of the following: (1) seropositivity for hepatitis B surface antigens or antibodies to hepatitis $\mathrm{C}$ virus, (2) excessive alcohol intake (>20 g/day), (3) other causes of liver disease, and (4) medications known to produce fatty liver disease.

US examination of the liver was performed by experienced radiologists who were unaware of the clinical information. The diagnosis of fatty liver was determined by ultrasonography (Acusion, Sequoia 512, Siemens, Mountain View, CA) using previously described standardized criteria [16].

2.4. Statistical Analysis. Comparisons of continuous variables between the two groups were performed with Student's $t$-test, and categorical variables were compared using a chisquare test or Fisher's exact test. Variables that were statistically significant by univariate analysis and known risk factors were added to a multiple logistic regression model to identify independent predictors of the presence of NAFLD. Statistical analysis was performed with SPSS 19.0 (SPSS Inc.; Chicago, IL, USA). $P$ values $<0.05$ were considered statistically to be significant.

\section{Results}

3.1. Baseline Characteristics. A total of 1,423 subjects were analyzed. The anthropometric, clinical, and laboratory characteristics of the subjects are shown in Table 1. Mean age was $52.4 \pm 9.4$ years. NAFLD was found in $294(20.7 \%)$ subjects. Among the total subjects, 695 women (48.9\%) were in menopausal state. Postmenopausal women had an older age, higher BMI, larger waist circumference, and higher levels of AST, ALT, GGT, fasting glucose, triglycerides, LDLcholesterol, and HOMA than premenopausal women $(P<$ 0.001). The presence of hypertension, diabetes, and MS was also higher in postmenopausal women compared to premenopausal women. The prevalence of NAFLD was higher in postmenopausal women than in premenopausal women (27.2\% versus $14.4 \%, P<0.001$, Table 1$)$.

In postmenopausal women, subjects with NAFLD had an older age, higher BMI, larger waist circumference, higher levels of AST, ALT, GGT, fasting glucose, triglycerides, and HOMA, and lower HDL-cholesterol than those without NAFLD $(P<0.001)$. The presence of hypertension, diabetes, MS, and NAFLD was higher in subjects with NAFLD compared to those without NAFLD. The rate of hormone replacement therapy was lower in subjects with NALFD than those without NAFLD (Table 2).

In premenopausal women, subjects with NAFLD had an older age, higher BMI, larger waist circumference, higher levels of AST, ALT, GGT, fasting glucose, triglycerides, LDL-cholesterol, and HOMA, and lower HDL-cholesterol than those without NAFLD $(P<0.001)$. The presence of hypertension, diabetes, MS, and NAFLD was higher in 
TABLE 1: Comparison of baseline characteristics in premenopausal women versus postmenopausal women.

\begin{tabular}{|c|c|c|c|}
\hline & Premenopausal women & Postmenopausal women & $P$ \\
\hline Number of subjects & 728 & 695 & $<0.001$ \\
\hline Age, years & $46.9 \pm 8.3$ & $58.2 \pm 6.7$ & $<0.001$ \\
\hline Body mass index, $\mathrm{kg} / \mathrm{m}^{2}$ & $21.6 \pm 2.7$ & $22.6 \pm 2.8$ & $<0.001$ \\
\hline Waist circumference, $\mathrm{cm}$ & $78.7 \pm 6.9$ & $82.5 \pm 7.4$ & $<0.001$ \\
\hline AST, IU/L & $19.6 \pm 6.7$ & $22.9 \pm 7.1$ & $<0.001$ \\
\hline ALT, IU/L & $17.2 \pm 9.9$ & $21.8 \pm 12.3$ & $<0.001$ \\
\hline GGT, IU/L & $19.3 \pm 14.7$ & $24.3 \pm 20.0$ & $<0.001$ \\
\hline Fasting glucose, $\mathrm{mg} / \mathrm{dL}$ & $90.5 \pm 10.7$ & $94.6 \pm 14.9$ & $<0.001$ \\
\hline Triglycerides, mg/dL & $84.4 \pm 56.4$ & $99.7 \pm 59.4$ & $<0.001$ \\
\hline HDL-cholesterol, mg/dL & $58.0 \pm 13.2$ & $51.0 \pm 10.7$ & 0.03 \\
\hline LDL-cholesterol, mg/dL & $117.7 \pm 27.9$ & $128.4 \pm 30.6$ & $<0.001$ \\
\hline CRP, mg/dL & $0.08 \pm 0.2$ & $0.11 \pm 0.24$ & 0.019 \\
\hline HOMA & $1.4 \pm 1.0$ & $1.6 \pm 1.3$ & 0.013 \\
\hline Smoking, \% & 2.8 & 2.2 & 0.291 \\
\hline Regular exercise, $\%$ & 38.2 & 19.6 & $<0.001$ \\
\hline Hypertension, \% & 17.2 & 36.2 & $<0.001$ \\
\hline Diabetes, \% & 12.1 & 23.7 & $<0.001$ \\
\hline Metabolic syndrome, \% & 8.8 & 25.9 & $<0.001$ \\
\hline NAFLD, $\%$ & 14.4 & 27.2 & $<0.001$ \\
\hline
\end{tabular}

Data are presented as the mean $\pm \mathrm{SD}$.

$P$ value by Student's $t$-test for continuous variables and chi-square test for categorical variables.

ALT: alanine aminotransferase; AST: aspartate aminotransferase; GGT: gamma-glutamyl transpeptidase; HDL: high density lipoprotein; LDL: low density lipoprotein; CRP: C-reactive protein; HOMA: homeostasis model assessment-estimated insulin resistance; NAFLD: nonalcoholic fatty liver disease.

TABLE 2: Comparison of baseline characteristics in postmenopausal women without NAFLD versus with NAFLD.

\begin{tabular}{|c|c|c|c|}
\hline & Postmenopausal women without NAFLD & Postmenopausal women with NAFLD & $P$ \\
\hline Number of subjects & 506 & 189 & \\
\hline Age, years & $57.7 \pm 6.6$ & $59.6 \pm 6.7$ & $<0.001$ \\
\hline Body mass index, $\mathrm{kg} / \mathrm{m}^{2}$ & $21.9 \pm 2.3$ & $24.5 \pm 2.9$ & $<0.001$ \\
\hline Waist circumference, $\mathrm{cm}$ & $80.8 \pm 6.8$ & $86.9 \pm 7.0$ & $<0.001$ \\
\hline AST, IU/L & $22.3 \pm 5.8$ & $24.6 \pm 9.4$ & $<0.001$ \\
\hline ALT, IU/L & $19.3 \pm 8.9$ & $28.3 \pm 17.0$ & $<0.001$ \\
\hline GGT, IU/L & $21.9 \pm 17.3$ & $30.9 \pm 24.8$ & $<0.001$ \\
\hline Fasting glucose, $\mathrm{mg} / \mathrm{dL}$ & $92.1 \pm 11.0$ & $101.5 \pm 20.5$ & $<0.001$ \\
\hline Triglycerides, mg/dL & $86.7 \pm 46.0$ & $134.4 \pm 75.4$ & $<0.001$ \\
\hline HDL-cholesterol, mg/dL & $60.7 \pm 12.0$ & $54.0 \pm 10.3$ & $<0.001$ \\
\hline LDL-cholesterol, mg/dL & $127.6 \pm 30.3$ & $130.4 \pm 31.3$ & 0.216 \\
\hline CRP, mg/dL & $0.09 \pm 0.2$ & $0.16 \pm 0.28$ & 0.006 \\
\hline HOMA & $1.4 \pm 1.2$ & $2.2 \pm 1.4$ & $<0.001$ \\
\hline Smoking, \% & 2.2 & 2.2 & 0.616 \\
\hline Regular exercise, \% & 17.4 & 25.9 & 0.012 \\
\hline Hypertension, \% & 30.1 & 52.4 & $<0.001$ \\
\hline Diabetes, $\%$ & 16.7 & 42.3 & $<0.001$ \\
\hline Metabolic syndrome, $\%$ & 16.8 & 50.3 & $<0.001$ \\
\hline Hormone therapy & $10.9 \%$ & $6.3 \%$ & 0.045 \\
\hline
\end{tabular}

Data are presented as the mean $\pm \mathrm{SD}$.

$P$ value by Student's $t$-test for continuous variables and chi-square test for categorical variables.

ALT: alanine aminotransferase; AST: aspartate aminotransferase; GGT: gamma-glutamyl transpeptidase; HDL: high density lipoprotein; LDL: low density lipoprotein; CRP: C-reactive protein; HOMA: homeostasis model assessment-estimated insulin resistance; NAFLD: nonalcoholic fatty liver disease. 
TABLE 3: Comparison of baseline characteristics in premenopausal women without NAFLD versus with NAFLD.

\begin{tabular}{|c|c|c|c|}
\hline & Premenopausal women without NAFLD & Premenopausal women with NAFLD & $P$ \\
\hline Number of subjects & 623 & 105 & \\
\hline Age, years & $46.3 \pm 8.0$ & $50.8 \pm 9.0$ & $<0.001$ \\
\hline Body mass index, $\mathrm{kg} / \mathrm{m}^{2}$ & $21.1 \pm 2.3$ & $24.6 \pm 3.3$ & $<0.001$ \\
\hline Waist circumference, $\mathrm{cm}$ & $77.5 \pm 6.2$ & $85.6 \pm 7.2$ & $<0.001$ \\
\hline AST, IU/L & $19.1 \pm 5.9$ & $22.7 \pm 9.7$ & $<0.001$ \\
\hline ALT, IU/L & $19.3 \pm 8.9$ & $28.3 \pm 17.0$ & $<0.001$ \\
\hline GGT, IU/L & $18.0 \pm 13.3$ & $27.3 \pm 19.4$ & $<0.001$ \\
\hline Fasting glucose, $\mathrm{mg} / \mathrm{dL}$ & $89.1 \pm 8.5$ & $98.9 \pm 17.0$ & $<0.001$ \\
\hline Triglycerides, mg/dL & $75.5 \pm 39.6$ & $137.2 \pm 97.8$ & $<0.001$ \\
\hline HDL-cholesterol, mg/dL & $61.7 \pm 12.3$ & $52.0 \pm 10.1$ & $<0.001$ \\
\hline LDL-cholesterol, mg/dL & $115.7 \pm 26.9$ & $129.6 \pm 30.7$ & $<0.001$ \\
\hline CRP, mg/dL & $0.07 \pm 0.2$ & $0.13 \pm 0.1$ & 0.012 \\
\hline HOMA & $1.2 \pm 0.6$ & $2.5 \pm 1.8$ & $<0.001$ \\
\hline Smoking, \% & 2.9 & 1.9 & 0.742 \\
\hline Regular exercise, $\%$ & 34.2 & 46.7 & 0.103 \\
\hline Hypertension, \% & 14.0 & 35.2 & $<0.001$ \\
\hline Diabetes, \% & 8.5 & 33.3 & $<0.001$ \\
\hline Metabolic syndrome, $\%$ & 5.5 & 28.6 & $<0.001$ \\
\hline
\end{tabular}

Data are presented as the mean \pm SD.

$P$ value by Student's $t$-test for continuous variables and chi-square test for categorical variables.

ALT: alanine aminotransferase; AST: aspartate aminotransferase; GGT: gamma-glutamyl transpeptidase; HDL: high density lipoprotein; LDL: low density lipoprotein; CRP: C-reactive protein; HOMA: homeostasis model assessment-estimated insulin resistance; NAFLD: nonalcoholic fatty liver disease.

subjects with NAFLD compared to those without NAFLD (Table 3).

3.2. Risk Factors for NALFD in Women. We performed a logistic regression analysis to determine the risk factors for NAFLD in women. Univariate analysis showed that a postmenopausal state increased the risk of NAFLD (odds ratio $2.02(1.42-2.86), P<0.001)$; however, the statistical significance disappeared after adjusting for other confounding factors. Multivariate analysis indicated that diabetes, triglycerides levels, central obesity, and ALT levels increased the risk of NAFLD (Table 4).

Next, we stratified the subjects according to menopausal state. In postmenopausal women, the presence of diabetes, triglycerides levels, and central obesity showed a significant association with risk for NAFLD; however, hormone replacement therapy was not a risk factor for the development of NAFLD (Table 5). In premenopausal women, low HDLcholesterol, central obesity, and HOMA showed a significant association with increased risk for NAFLD after multivariate analysis (Table 6).

\section{Discussion}

The study identified different metabolic factors for NAFLD in women in relation to menopause status; diabetes, triglycerides, and central obesity were risk factors in postmenopausal women, while low HDL-cholesterol, central obesity, and HOMA were risk factors in those with premenopausal status. However, in both cases key issues are the same: central obesity and insulin resistance. These results suggested that there is a close link between NAFLD and components of MS. Interestingly, the presence of menopause showed no significant association with increased risk of NAFLD, and hormone replacement therapy showed no association with NAFLD in postmenopausal women.

The prevalence of NAFLD in the current study (20.7\%) was slightly higher than the prevalence $(10-15 \%)$ reported in previous studies of Asian women [10, 17, 18]. However, the prevalence of NAFLD in postmenopausal women in this study $(27.2 \%)$ was at the average compared to those in previous studies (ranging from $15 \%$ to $57 \%$ ) $[12,17,19]$. These variable results between different studies may be due to different populations heterogeneously selected and the contributing various factors to the development of NAFLD in postmenopausal women.

Metabolic syndrome is a cluster of metabolic factors that are related to increased cardiovascular risks. The associations between NAFLD and MS have been well established [4, 20]. Obesity, hyperlipidemia, and type 2 diabetes, which are components of MS, are frequently coexisting conditions in subjects with NAFLD [21]. The incidence of MS was higher in subjects with NAFLD than those without NAFLD $(33.8 \%$ versus $10.6 \%$ ) [22]. In a Brazilian study on postmenopausal women, more than $90 \%$ of the subjects with NAFLD had MS [19]. Consistent with previous results, more than $50 \%$ of postmenopausal women with NAFLD had MS and multiple components of MS showed an association with NAFLD in our study. Among the various components of MS, central obesity was revealed as a key risk factor of NAFLD in both pre- and postmenopausal women, suggesting a critical role in the pathogenesis of NAFLD. In agreement with our results, 
TABLE 4: Unadjusted and adjusted analyses for the risk of NAFLD in all subjects.

\begin{tabular}{|c|c|c|c|c|}
\hline & Unadjusted OR (95\% CI) & $P$ & Adjusted OR (95\% CI) & $P$ \\
\hline Age & $1.05(1.03-1.07)$ & $<0.001$ & $1.00(0.97-1.03)$ & 0.989 \\
\hline Postmenopause & $2.02(1.42-2.86)$ & $<0.001$ & $1.07(0.64-1.79)$ & 0.803 \\
\hline Hypertension & $2.97(2.09-4.21)$ & $<0.001$ & $1.52(0.96-2.38)$ & 0.073 \\
\hline Diabetes & $4.52(3.08-6.64)$ & $<0.001$ & $2.35(1.40-3.95)$ & 0.001 \\
\hline Triglyceridemia & $6.47(4.12-10.18)$ & $<0.001$ & $3.22(1.81-5.72)$ & $<0.001$ \\
\hline HDL & $0.33(0.23-0.48)$ & $<0.001$ & $0.60(0.38-0.97)$ & 0.036 \\
\hline Central obesity & $6.45(4.48-9.27)$ & $<0.001$ & $3.67(2.36-5.70)$ & $<0.001$ \\
\hline Smoking & $0.93(0.34-2.53)$ & 0.891 & $0.75(0.23-2.46)$ & 0.628 \\
\hline Exercise & $1.43(1.00-2.05)$ & 0.052 & $1.56(0.99-2.47)$ & 0.057 \\
\hline ALT & $1.08(1.06-1.10)$ & $<0.001$ & $1.07(1.04-1.09)$ & $<0.001$ \\
\hline GGT & $1.02(1.01-1.03)$ & $<0.001$ & $1.00(0.99-1.01)$ & 0.353 \\
\hline HOMA & $2.33(1.91-2.54)$ & $<0.001$ & $1.23(0.97-1.56)$ & 0.093 \\
\hline
\end{tabular}

NAFLD: nonalcoholic fatty liver disease; ALT: alanine aminotransferase; GGT: gamma-glutamyl transpeptidase; OR: odds ratio; CI: confidence interval; HDL: high density lipoprotein; HOMA: homeostasis model assessment-estimated insulin resistance.

TABLE 5: Unadjusted and adjusted analyses for the risk of NAFLD in postmenopausal women.

\begin{tabular}{|c|c|c|c|c|}
\hline & Unadjusted OR (95\% CI) & $P$ & Adjusted OR (95\% CI) & $P$ \\
\hline Age & $1.03(0.99-1.06)$ & 0.124 & $0.97(0.93-1.01)$ & 0.179 \\
\hline Hypertension & $2.68(1.73-4.17)$ & $<0.001$ & $1.50(0.86-2.61)$ & 0.152 \\
\hline Diabetes & $3.57(2.22-5.74)$ & $<0.001$ & $2.38(1.30-4.37)$ & 0.005 \\
\hline Triglyceridemia & $5.57(3.09-10.03)$ & $<0.001$ & $4.51(2.16-9.43)$ & $<0.001$ \\
\hline $\mathrm{HDL}$ & $0.46(0.29-0.75)$ & 0.002 & $0.85(0.46-1.57)$ & 0.592 \\
\hline Central obesity & $4.91(3.10-7.76)$ & $<0.001$ & $4.17(2.40-7.23)$ & $<0.001$ \\
\hline Hormone therapy & $0.03(0.13-0.88)$ & 0.026 & $0.47(0.15-1.44)$ & 0.185 \\
\hline Smoking & $0.78(0.21-2.89)$ & 0.713 & $0.56(0.13-2.45)$ & 0.439 \\
\hline Exercise & $1.59(0.96-2.62)$ & 0.072 & $1.35(0.74-2.48)$ & 0.332 \\
\hline ALT & $1.07(1.04-1.10)$ & $<0.001$ & $1.07(1.04-1.10)$ & $<0.001$ \\
\hline GGT & $1.01(1.00-1.02)$ & 0.022 & $0.99(0.98-1.00)$ & 0.122 \\
\hline HOMA & $1.72(1.37-2.16)$ & $<0.001$ & $1.02(0.84-1.24)$ & 0.838 \\
\hline
\end{tabular}

NAFLD: nonalcoholic fatty liver disease; ALT: alanine aminotransferase; GGT: gamma-glutamyl transpeptidase; OR: odds ratio; CI: confidence interval; HDL: high density lipoprotein; HOMA: homeostasis model assessment-estimated insulin resistance.

TABLE 6: Unadjusted and adjusted analyses for the risk of NAFLD in premenopausal women.

\begin{tabular}{|c|c|c|c|c|}
\hline & Unadjusted OR (95\% CI) & $P$ & Adjusted OR (95\% CI) & $P$ \\
\hline Age & $1.03(0.99-1.06)$ & 0.124 & $1.04(0.99-1.10)$ & 0.121 \\
\hline Hypertension & $2.68(1.73-4.17)$ & $<0.001$ & $1.57(0.69-3.55)$ & 0.283 \\
\hline Diabetes & $3.57(2.22-5.74)$ & $<0.001$ & $2.07(0.77-5.53)$ & 0.149 \\
\hline Triglyceridemia & $5.57(3.09-10.03)$ & $<0.001$ & $1.75(0.62-5.00)$ & 0.293 \\
\hline HDL & $0.46(0.29-0.75)$ & 0.002 & $0.36(0.16-0.83)$ & 0.016 \\
\hline Central obesity & $4.91(3.10-7.76)$ & $<0.001$ & $2.79(1.25-6.24)$ & 0.012 \\
\hline Smoking & $0.78(0.21-2.89)$ & 0.713 & $0.70(0.08-6.14)$ & 0.744 \\
\hline Exercise & $1.59(0.96-2.62)$ & 0.072 & $1.70(0.79-3.66)$ & 0.176 \\
\hline ALT & $1.07(1.04-1.10)$ & $<0.001$ & $1.06(1.02-1.10)$ & 0.003 \\
\hline GGT & $1.01(1.00-1.02)$ & 0.022 & $1.01(0.99-1.03)$ & 0.475 \\
\hline HOMA & $1.72(1.37-2.16)$ & $<0.001$ & $2.46(1.51-4.00)$ & $<0.001$ \\
\hline
\end{tabular}

NAFLD: nonalcoholic fatty liver disease; ALT: alanine aminotransferase; GGT: gamma-glutamyl transpeptidase; OR: odds ratio; CI: confidence interval; HDL: high density lipoprotein; HOMA: homeostasis model assessment-estimated insulin resistance. 
visceral fat accumulation showed a positive correlation with hepatic steatosis $[23,24]$.

The prevalence of NAFLD was higher in postmenopausal women than premenopausal women in this study. Previous studies indicated that the menopausal status may influence the prevalence of NAFLD, suggesting the association with hepatic steatosis [8-11]. There have been several studies that have investigated the influence of estrogen on NAFLD. An animal study using the aromatase-knockout mouse showed that hepatic steatosis developed in aromatase-deficient mice $[25,26]$, and estradiol replacement therapy reversed hepatic steatosis, suggesting a role for estrogen in maintaining lipid homeostasis in the liver [27]. Previous human studies regarding a potent antagonist of estrogen, tamoxifen, showed that tamoxifen induced and progressed steatohepatitis in the treatment of breast cancer [28, 29]. A randomized controlled trial with tamoxifen performed in Italy showed that tamoxifen was associated with an increased risk of development of nonalcoholic steatohepatitis only in overweight and obese women [30]. Although the protective effect of estrogen in hepatic steatosis or NASH is distinct, the presence of menopause and hormone replacement therapy in postmenopausal women were not risk factors for NAFLD in this study, while metabolic risk factors had significant associations with an increased risk of NAFLD. The lack of association between menopause and NAFLD might be explained by potential mechanisms like the following. (i) Postmenopausal women have deficiencies in estrogen and relative androgen excess, which might lead to redistribution of total body fat such as increase of visceral fat, which might cause the development of insulin resistance [31]. Estrogen deficiency by itself is not as important as the relationship between testosterone and estradiol and exogenous administration of androgens to women has been demonstrated to insulin resistance [3133], and clinical trials of postmenopausal women have not demonstrated benefits of exogenous estradiol [34-36]. In the same context, increased prevalence of NAFLD in patients with polycystic ovary syndrome is explained by androgen excess. Central adiposity and insulin resistance are the main factors related to NAFLD in postmenopausal women. (ii) In addition, multiple confounders such as metabolic factors, inflammation, nutritional factors, and age might influence the development of NALFD in postmenopausal women [9]. In this study, metabolic factors were associated significantly with NAFLD rather than menopause status. (iii) Older women have decreased physical activity and greater adiposity [37, 38], which may influence the changes in body composition and metabolic profiles. Consistent to our results, Hamaguchi et al. [17] indicated that weight gain and MS were independent risk factors for increased risk of NAFLD in both pre- and postmenopausal women; however, a postmenopausal state and hormone replacement therapy had no significant association with risk for NAFLD. Codes et al. showed that there was no difference in the frequency or severity of steatosis in relation to hormone replacement therapy in women with chronic hepatitis C [39].

A strength of this study is the determination of different metabolic factors in women with pre- or postmenopausal state. In addition, the subjects in this study are regarded to be representative of the general population due to the nature of being recruited at a health check-up visit. However, there were several limitations in the study. First, the design of a cross-sectional study makes it difficult to evaluate the temporal association between NAFLD and metabolic risk factors. Second, we could not obtain liver histology results, the gold standard diagnosis for NAFLD. US can introduce false-negative results when fatty infiltration is below $30 \%$ [40]. However, it was impossible to perform an invasive test in an apparently healthy population, and US is used as the first-line method for clinical practical guidelines [41, 42]. Third, information on dietary components and tests for inflammation were unavailable.

In conclusion, our findings showed different metabolic factors for NAFLD in pre- and postmenopausal women. However, the key issues are the same: central obesity and insulin resistance. These results reemphasize the importance of metabolic factors irrespective of menopausal status in the pathogenesis of NAFLD in women.

\section{Abbreviations}

$\begin{array}{ll}\text { ALT: } & \text { Alanine aminotransferase } \\ \text { BMI: } & \text { Body mass index } \\ \text { HDL: } & \text { High density lipoprotein } \\ \text { MS: } & \text { Metabolic syndrome } \\ \text { NAFLD: } & \text { Nonalcoholic fatty liver disease } \\ \text { AST: } & \text { Aspartate aminotransferase } \\ \text { GGT: } & \text { Gamma-glutamyl transpeptidase } \\ \text { HOMA: } & \text { Homeostasis model assessment-estimated } \\ & \text { insulin resistance } \\ \text { US: } & \text { Ultrasonography } \\ \text { LDL: } & \text { Low density lipoprotein. }\end{array}$

\section{Conflict of Interests}

The authors declare that there is no conflict of interests regarding the publication of this paper.

\section{References}

[1] G. Vernon, A. Baranova, and Z. M. Younossi, "Systematic review: the epidemiology and natural history of non-alcoholic fatty liver disease and non-alcoholic steatohepatitis in adults," Alimentary Pharmacology and Therapeutics, vol. 34, no. 3, pp. 274-285, 2011.

[2] D. M. Torres, C. D. Williams, and S. A. Harrison, "Features, diagnosis, and treatment of nonalcoholic fatty liver disease," Clinical Gastroenterology and Hepatology, vol.10, no. 8, pp. 837858, 2012.

[3] J. M. Clark, "The epidemiology of nonalcoholic fatty liver disease in adults," Journal of Clinical Gastroenterology, vol. 40, supplement 1, pp. S5-S10, 2006.

[4] G. Marchesini, M. Brizi, G. Blanchi et al., "Nonalcoholic fatty liver disease: a feature of the metabolic syndrome," Diabetes, vol. 50, no. 8, pp. 1844-1850, 2001.

[5] G. Pagano, G. Pacini, G. Musso et al., "Nonalcoholic steatohepatitis, insulin resistance, and metabolic syndrome: further evidence for an etiologic association," Hepatology, vol. 35, no. 2, pp. 367-372, 2002. 
[6] G. Marchesini, E. Bugianesi, G. Forlani et al., "Nonalcoholic fatty liver, steatohepatitis, and the metabolic syndrome," Hepatology, vol. 37, no. 4, pp. 917-923, 2003.

[7] J. C. Lovejoy, C. M. Champagne, L. de Jonge, H. Xie, and S. R. Smith, "Increased visceral fat and decreased energy expenditure during the menopausal transition," International Journal of Obesity, vol. 32, no. 6, pp. 949-958, 2008.

[8] A. Suzuki and M. F. Abdelmalek, "Nonalcoholic fatty liver disease in women," Women's Health, vol. 5, no. 2, pp. 191-203, 2009.

[9] H. Völzke, S. Schwarz, S. E. Baumeister et al., "Menopausal status and hepatic steatosis in a general female population," Gut, vol. 56, no. 4, pp. 594-595, 2007.

[10] S. H. Park, W. K. Jeon, S. H. Kim et al., "Prevalence and risk factors of non-alcoholic fatty liver disease among Korean adults," Journal of Gastroenterology and Hepatology, vol. 21, no. 1, pp. 138-143, 2006.

[11] J. D. Yang, M. F. Abdelmalek, H. Pang et al., "Gender and menopause impact severity of fibrosis among patients with nonalcoholic steatohepatitis," Hepatology, vol. 59, no. 4, pp. 1406-1414, 2014.

[12] Y. Gutierrez-Grobe, G. Ponciano-Rodríguez, M. H. Ramos, M. Uribe, and N. Méndez-Sánchez, "Prevalence of non alcoholic fatty liver disease in premenopausal, posmenopausal and polycystic ovary syndrome women. The role of estrogens," Annals of Hepatology, vol. 9, no. 4, pp. 402-409, 2010.

[13] J. McKenzie, B. M. Fisher, A. J. Jaap, A. Stanley, K. Paterson, and N. Sattar, "Effects of HRT on liver enzyme levels in women with type 2 diabetes: a randomized placebo-controlled trial," Clinical Endocrinology, vol. 65, no. 1, pp. 40-44, 2006.

[14] P. W. F. Wilson, R. B. D’Agostino, D. Levy, A. M. Belanger, H. Silbershatz, and W. B. Kannel, "Prediction of coronary heart disease using risk factor categories," Circulation, vol. 97, no. 18, pp. 1837-1847, 1998.

[15] "Executive summary of the third report of the National Cholesterol Education Program (NCEP) expert panel on detection, evaluation, and treatment of high blood cholesterol in adults (adult treatment panel III)," The Journal of the American Medical Association, vol. 285, no. 19, pp. 2486-2497, 2001.

[16] S.-Y. Choi, D. Kim, H. J. Kim et al., "The relation between non-alcoholic fatty liver disease and the risk of coronary heart disease in Koreans," The American Journal of Gastroenterology, vol. 104, no. 8, pp. 1953-1960, 2009.

[17] M. Hamaguchi, T. Kojima, A. Ohbora, N. Takeda, M. Fukui, and T. Kato, "Aging is a risk factor of nonalcoholic fatty liver disease in premenopausal women," World Journal of Gastroenterology, vol. 18, no. 3, pp. 237-243, 2012.

[18] J. C. Bae, Y. K. Cho, W. Y. Lee et al., "Impact of nonalcoholic fatty liver disease on insulin resistance in relation to HbAlc levels in nondiabetic subjects," The American Journal of Gastroenterology, vol. 105, no. 11, pp. 2389-2395, 2010.

[19] S. de Souza Bruno, M. H. Rodrigues, M. C. B. Alvares, J. NahasNeto, and E. A. P. Nahas, "Non-alcoholic fatty liver disease and its associated risk factors in Brazilian postmenopausal women," Climacteric, vol. 17, no. 4, pp. 465-471, 2014.

[20] G. Tarantino, G. Saldalamacchia, P. Conca, and A. Arena, "Nonalcoholic fatty liver disease: further expression of the metabolic syndrome," Journal of Gastroenterology and Hepatology, vol. 22, no. 3, pp. 293-303, 2007.

[21] J. B. Dixon, P. S. Bhathal, and P. E. O'Brien, "Nonalcoholic fatty liver disease: predictors of nonalcoholic steatohepatitis and liver fibrosis in the severely obese," Gastroenterology, vol. 121, no. 1, pp. 91-100, 2001.

[22] Z.-W. Chen, L.-Y. Chen, H.-L. Dai, J.-H. Chen, and L.-Z. Fang, "Relationship between alanine aminotransferase levels and metabolic syndrome in nonalcoholic fatty liver disease," Journal of Zhejiang University: Science B, vol. 9, no. 8, pp. 616622, 2008.

[23] Y. Eguchi, T. Eguchi, T. Mizuta et al., "Visceral fat accumulation and insulin resistance are important factors in nonalcoholic fatty liver disease," Journal of Gastroenterology, vol. 41, no. 5, pp. 462-469, 2006.

[24] B. J. Park, Y. J. Kim, D. H. Kim et al., "Visceral adipose tissue area is an independent risk factor for hepatic steatosis," Journal of Gastroenterology and Hepatology, vol. 23, no. 6, pp. 900-907, 2008.

[25] M. E. E. Jones, A. W. Thorburn, K. L. Britt et al., "Aromatasedeficient (ArKO) mice have a phenotype of increased adiposity," Proceedings of the National Academy of Sciences of the United States of America, vol. 97, no. 23, pp. 12735-12740, 2000.

[26] K. N. Hewitt, W. C. Boon, Y. Murata, M. E. E. Jones, and E. R. Simpson, "The aromatase knockout mouse presents with a sexually dimorphic disruption to cholesterol homeostasis," Endocrinology, vol. 144, no. 9, pp. 3895-3903, 2003.

[27] Y. Nemoto, K. Toda, M. Ono et al., "Altered expression of fatty acid-metabolizing enzymes in aromatase-deficient mice," The Journal of Clinical Investigation, vol. 105, no. 12, pp. 1819-1825, 2000.

[28] M. van Hoof, J. Rahier, and Y. Horsmans, “Tamoxifen-induced steatohepatitis," Annals of Internal Medicine, vol. 124, no. 9, pp. 855-856, 1996.

[29] K. A. Oien, D. Moffat, G. W. Curry et al., "Cirrhosis with steatohepatitis after adjuvant tamoxifen," The Lancet, vol. 353, no. 9146, pp. 36-37, 1999.

[30] S. Bruno, P. Maisonneuve, P. Castellana et al., "Incidence and risk factors for non-alcoholic steatohepatitis: prospective study of 5408 women enrolled in Italian tamoxifen chemoprevention trial," The British Medical Journal, vol. 330, no. 7497, pp. 932-937, 2005.

[31] J. I. Torréns, K. Sutton-Tyrrell, X. Zhao et al., "Relative androgen excess during the menopausal transition predicts incident metabolic syndrome in midlife women: study of Women's Health Across the Nation," Menopause, vol. 16, no. 2, pp. 257264, 2009.

[32] G. D. Braunstein, "Androgen insufficiency in women: summary of critical issues," Fertility and Sterility, vol. 77, supplement 4, pp. S94-S99, 2002.

[33] K. H. Polderman, L. J. G. Gooren, H. Asscheman, A. Bakker, and R. J. Heine, "Induction of insulin resistance by androgens and estrogens," The Journal of Clinical Endocrinology \& Metabolism, vol. 79, no. 1, pp. 265-271, 1994.

[34] D. Grady, D. Herrington, V. Bittner et al., "Cardiovascular disease outcomes during 6.8 years of hormone therapy: heart and estrogen/progestin replacement study follow-up (HERS II)," The Journal of the American Medical Association, vol. 288, no. 1, pp. 49-57, 2002.

[35] J. E. Rossouw, G. L. Anderson, R. L. Prentice et al., "Risks and benefits of estrogen plus progestin in healthy postmenopausal women: principal results from the Women's Health Initiative randomized controlled trial," The Journal of the American Medical Association, vol. 288, pp. 321-333, 2002.

[36] G. L. Anderson, M. Limacher, A. R. Assaf et al., "Effects of conjugated equine estrogen in postmenopausal women with 
hysterectomy: the women's health initiative randomized controlled trial," The Journal of the American Medical Association, vol. 291, no. 14, pp. 1701-1712, 2004.

[37] F. Sun, I. J. Norman, and A. E. While, "Physical activity in older people: a systematic review," BMC Public Health, vol. 13, no. 1, article 449, 2013.

[38] R. J. Valentine, M. M. Misic, K. S. Rosengren, J. A. Woods, and E. M. Evans, "Sex impacts the relation between body composition and physical function in older adults," Menopause, vol. 16, no. 3, pp. 518-523, 2009.

[39] L. Codes, T. Asselah, D. Cazals-Hatem et al., "Liver fibrosis in women with chronic hepatitis C: evidence for the negative role of the menopause and steatosis and the potential benefit of hormone replacement therapy," Gut, vol. 56, no. 3, pp. 390-395, 2007.

[40] A. J. Sanyal, "AGA technical review on nonalcoholic fatty liver disease," Gastroenterology, vol. 123, no. 5, pp. 1705-1725, 2002.

[41] N. Chalasani, Z. Younossi, J. E. Lavine et al., "The diagnosis and management of non-alcoholic fatty liver disease: practice guideline by the American Gastroenterological Association, American Association for the Study of Liver Diseases, and American College of Gastroenterology," Gastroenterology, vol. 142, pp. 1592-1609, 2012.

[42] Korean Association for the Study of the Liver (KASL), "KASL clinical practice guidelines: management of nonalcoholic fatty liver disease," Clinical and Molecular Hepatology, vol. 19, pp. 325-348, 2013. 


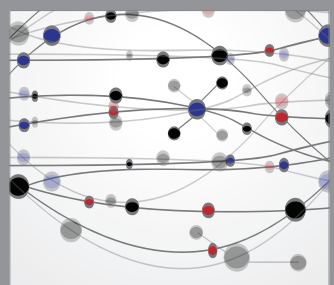

The Scientific World Journal
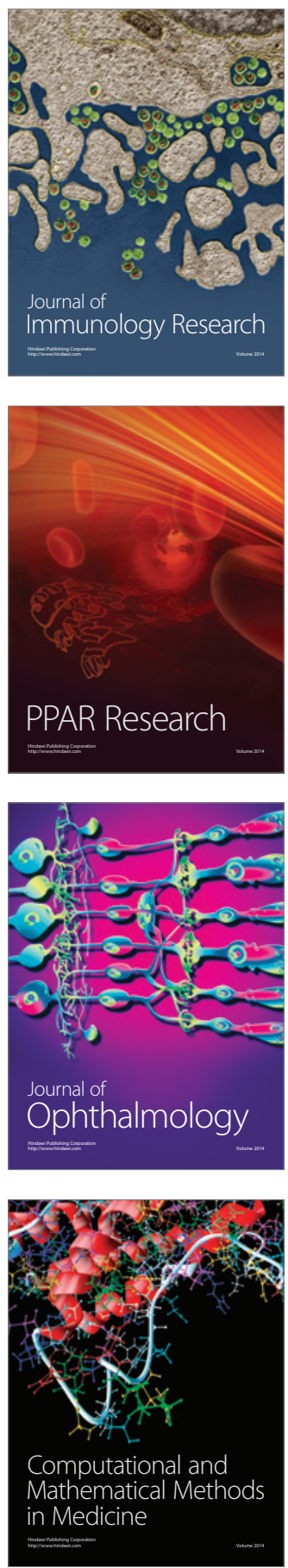

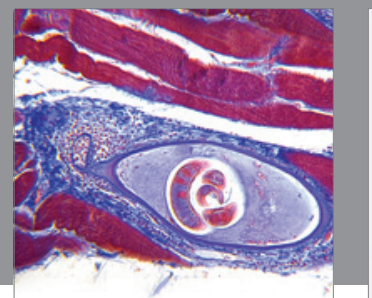

Gastroenterology

Research and Practice
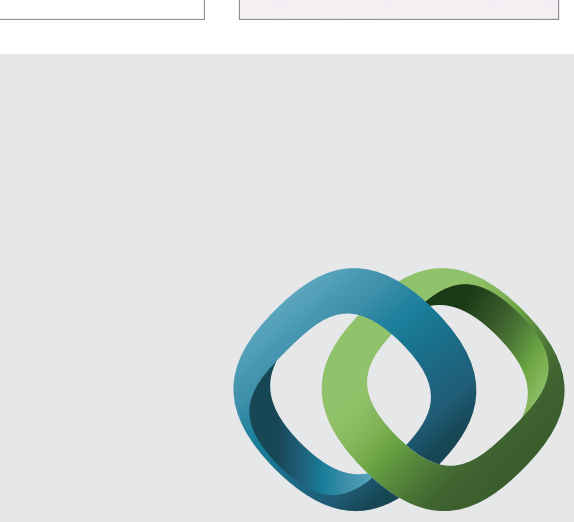

\section{Hindawi}

Submit your manuscripts at

http://www.hindawi.com
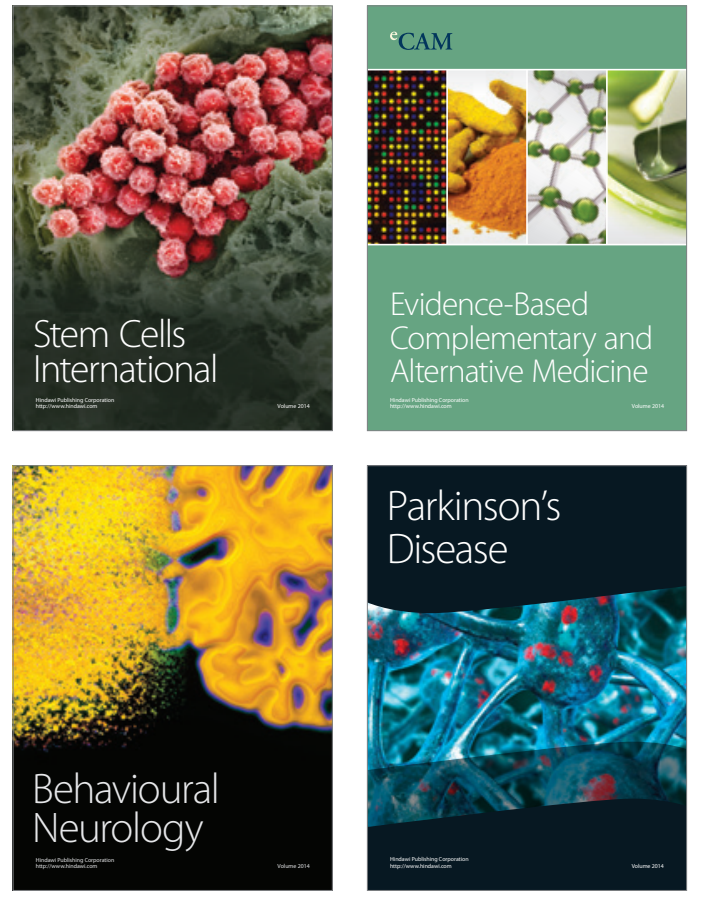
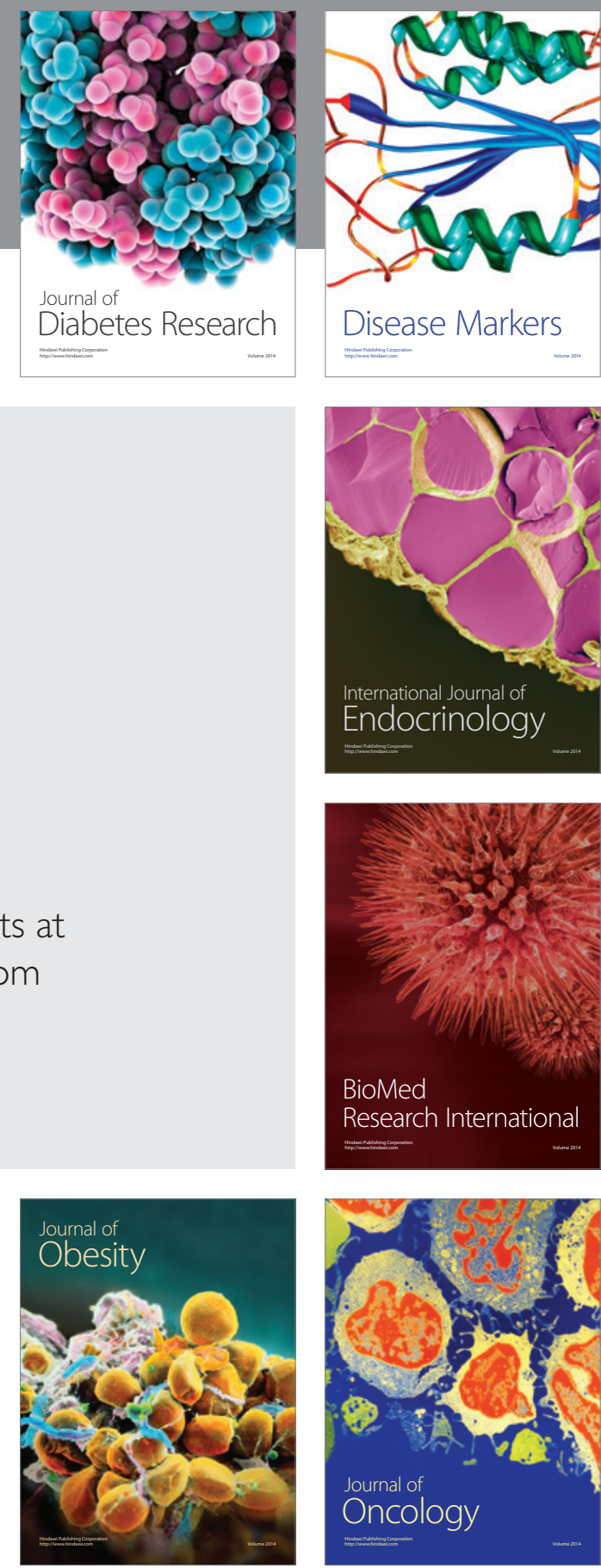

Disease Markers
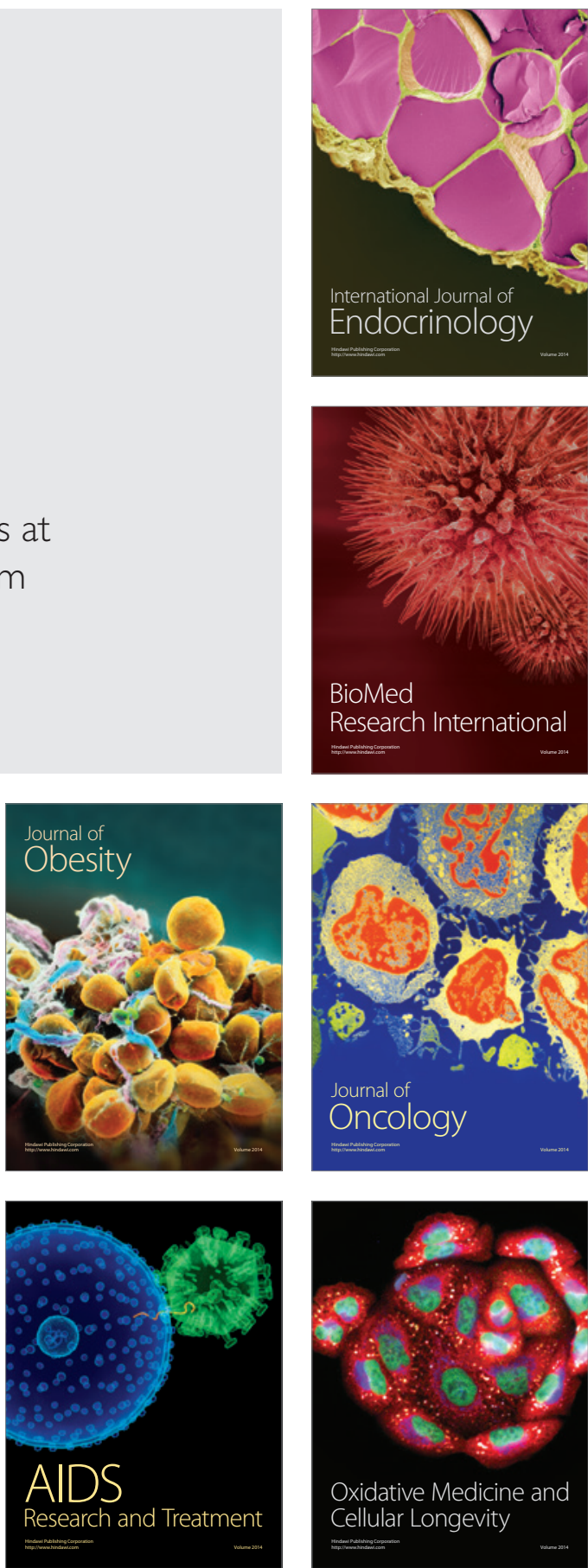